\title{
SISTEMA ANTI COLISIONES PARA NAVEGACIÓN EN INTERIORES DE UN QUACOPTER USANDO UN CONTROLADOR DIFUSO Y SENSORES DE RANGO
}

\author{
ANTI-COLLISION SYSTEM FOR INDOORS \\ QUADCOPTER NAVIGATION USING FUZZY \\ CONTROLLERS AND RANGE SENSORS
}

Rubén Darío Fonnegra Tarazona ${ }^{1}$, Felipe Ríos Lopera ${ }^{2}$, Germán David Góez Sánchez ${ }^{3}$

${ }^{1 .}$ Estudiante Ingeniería en Electrónica Instituto Tecnológico Metropolitano - ITM Medellín, Colombia

2. Estudiante Ingeniería en Electrónica Instituto Tecnológico Metropolitano - ITM Medellín, Colombia

${ }^{3}$. Docente Ocasional, Grupo Automática, Electrónica y Ciencias Computacionales Instituto Tenológico Metropolitano - ITM Medellín, Colombia

Recibido: 10Febrero de 2015

Aceptado: 6 Febrero de 2016

*Correspondencia del autor: Rubén Darío Fonnegra Tarazona; E-mail: rubenfonnegrat1015@gmail.com

\section{RESUMEN}

Este proyecto presenta los resultados de la introducción de un control de velocidad mediante la utilización de sensores de ultrasonido y acelerómetro para la detección de objetos, asociados a un controlador difuso para prevenir colisiones en espacios cerrados. Con la implementación de este modelo anti colisión, la navegación de robots aéreos en interiores se hace más segura.

Palabras claves: Sensores de rango, navegación espacial, evación de obstáculos, procesamiento de señales, sonar, Quadrotor, lógica difusa, controlador difuso.

\begin{abstract}
This project presents the results of introducing a speed control by using ultrasound and accelerometer sensors to detect objects associated to a fuzzy controller to prevent collisions in enclosed spaces. By implementing this anticollision model, indoor navigation of aerial robots becomes safer.
\end{abstract}

Keywords: range sensors, spatial navigation, obstacle avoidance, signal processing, sonar, Quadrotor, fuzzy logic, fuzzy controller 


\section{INTRODUCCIÓN}

La necesidad de controlar sistemas aéreos autónomos de forma remota ha generado una creciente inquietud en la investigación relacionada. La selección de un Estos vehículos comúnmente denominados como UAV (Unmanned Aerial Vehicle por sus siglas en ingles), depende del estudio del entorno de trabajo y de la aplicación que se le pretenda dar. La razón es que el UAV difiere enormemente dependiendo de la situación (1); esto incluye los posibles sensores a utilizar y el hardware y software a ser implementado (2), diferenciándose en tres tamaños básicos: Grandes, medianos y pequeños, caracterizados por el tipo de motores; si son eléctricos, a reacción o combustión y la autonomía de vuelo.

Los UAV de tamaño grande y mediano se caracterizan por ser de ala fija, con autonomía de vuelo en horas, siendo el Predator y el Global Hawk vehículos aéreos de amplio reconocimiento en el ámbito militar por su uso en diferentes lugares del mundo. Su autonomía es de 36 horas y 16 horas de vuelo respectivamente (2), con guiado por un controlador humano de forma remota. Sus sistemas de reconocimiento se componen de un radar de apertura sintética, complementados por lentes ópticas y de infrarrojos. Para la navegación cuentan con sistemas GPS de alta precisión y altímetro, asociados a un sistema de comunicación satelital con el cual se realiza el monitoreo y control (3) (4).

Los UAV de tamaño reducido, son modelos de helicópteros, desde una, hasta varias aspas (tricopter, quadcopter u optocopter) siguen el mismo patrón de vuelo y control. Estos tipos de UAV no tienen aspas de cola para controlar la rotación, sino que en el caso de los quadcopter, dos motores rotarán en un sentido horario y los otros dos motores girarán en sentido anti

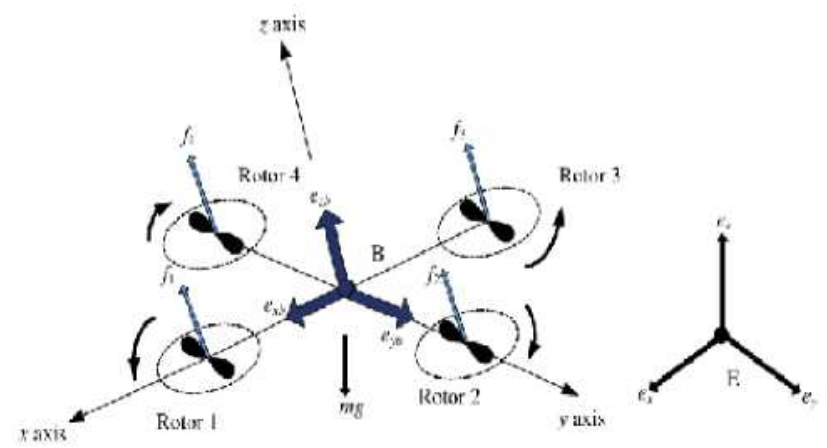

Figura 1, sistema de fuerzas y torque en quadroptor (9) horario, con la finalidad que el quadcopter compense la rotación y se mantenga estable (5); aunque dependiendo de la configuración pueden tener más motores, los cuales pueden ser controlados a partir de técnicas de control clásico (6) (7) o inteligencia artificial (8). En la figura 1, se muestra el sistema de fuerzas que interviene en un UAV tipo quadcopter.

Un quadrotor se controla variando únicamente la velocidad angular de los cuatro motores:

$x=(x, y, z)$ es el vector de posición del quadrotor

$\alpha=(\phi, \theta, \Psi)$ son los ángulos de Euler del quadrotor (pitch, roll y yaw respectivamente)(6).

En (9) se presenta un algoritmo de control basado en un sistema 3D, para navegación en interiores, donde el algoritmo de control es probado mediante simulación. Pero los métodos de control y navegación requieren información exacta de la posición a partir de las mediciones realizadas con giroscopio, acelerómetro y otros dispositivos de medición, ya sean sensores de distancia o geo-posicionados (10). En (11), presentan una técnica de posicionamiento en un entorno cerrado basados en sistemas de medición de distancia el cual se vale de un sistema distribuido para evitar colisiones.

Para determinar y resolver el sistema de navegación inercial (INS), se usan sensores que determinen la inclinación, lo mismo que sensores que entreguen la aceleración en tres ejes (x, y, z). Debido a la integración de los valores de aceleración de la posición se calcula con el aumento de error (la desventaja de todos los INS). Estos valores entregan la posición real estimada al controlador, donde se determina la señal de control a los motores basada en una modulación por ancho de pulso (PWM) (12). Otra técnica para navegación en interiores se observa en (13), donde se construye un sistema distribuido de sensores, no a bordo del UAV que le proporcionan información de la localización mediante un sistema de comunicación inalámbrica. Las pruebas realizadas muestran precisión de $50 \mathrm{~cm}$ en interiores, logrando descender de forma suave sin ayuda de un controlador humano.

\section{METODOS Y PROCEDIMIENTOS}

A continuación se describe como se construyeron los diferentes experimentos que permitieron obtener los 
datos de distancia y aceleración en cada uno de los ejes del UAV.

\section{A. Obtención de las mediciones de distancia}

Los sistemas mecánicos tienen frecuencias naturales de vibración. Cuando se excita un sistema mecánico en una de sus frecuencias naturales de oscilación, hay una transferencia máxima de energía por parte de la fuente excitadora hacia el sistema, y la amplitud de la vibración aumenta hasta un máximo. En estas condiciones decimos que el sistema está en resonancia con la fuente y nos referimos a la frecuencia particular en la cual esto ocurre como frecuencia de resonancia. La relación entre la frecuencia $\mathrm{f}$, la longitud de $\mathrm{La}$ relación entre la frecuencia $\mathrm{f}$, la longitud de onda $\lambda, \mathrm{y}$ la velocidad $\mathrm{v}$ de la onda, que se propaga a través del sistema es:

$$
v=\lambda * f
$$

Si se conoce la frecuencia y la longitud de onda, es posible deducir su velocidad; o si es conocida la longitud de onda y la velocidad, se puede calcular la frecuencia. Esto es fácilmente deducible en un sistema ideal, pero en realidad la velocidad del sonido está directamente relacionada con la temperatura ambiente y el material por el que se propague. Por esta razón se hace necesario implementar una técnica de corrección por temperatura ya que el sensor seleccionado de referencia HC-SR04 entrega la medida de tiempo de vuelo de la señal ultrasónica sin presentar corrección por temperatura, lo que es crítico en la navegación en interiores. En la figura 2, se presenta el diagrama de tiempos para este sensor.

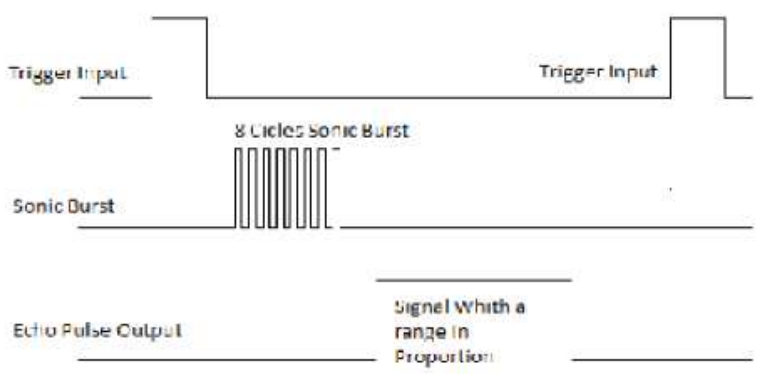

Figura 2, diagrama de tiempos del sensor hc-sr04

\section{B. Muestreo del Sensor Ultra Sonido}

Para garantizar el éxito del programa de muestreo se recogieron un número de muestras representativo a diferentes horas del día, contando con la presencia de un termómetro con el cual se registró la temperatura al momento tomar la muestra. El sensor ultrasonido entrega el valor del tiempo de vuelo de la señal de sonido. Aplicando la siguiente formula se puede determinar la velocidad del sonido en ese preciso momento.

$$
s=v_{\text {sonido }} * \frac{t_{\text {sonido }}}{2}
$$

$$
\begin{array}{ll}
\text { - } & s=\text { Distancia al objeto } \\
\text { - } & v=\text { Velocidad del sonido } \\
& t=\text { Tiempo de vuelo de la señal en segundos }
\end{array}
$$

La frecuencia de recolección de la muestra es la siguiente:

- $\quad 7$ Muestras, a una hora distinta
- $\quad 30$ Datos por muestra
Distancia $2 \mathrm{mts}$

La recolección de estas muestras se realizara en un total de tres semanas, con el fin de garantizar la homogeneidad de la muestra. En la tabla 1 se muestran los resultados estadísticos de las muestras en función de la velocidad del sonido a diferentes temperaturas.

\section{Estimación de la velocidad a partir de los sensores ultrasonido}

En la ecuación 3, se obtiene la velocidad del UAV tomando como la razón de cambio en la distancia recorrida desde un punto inicial al punto final. Estos puntos se determinan con la lectura de distancia desde el sensor ultrasonido hasta el objeto que se muestra como obstáculo en la dirección del movimiento (ut), con un intervalo de $160 \mathrm{~ms}$ entre las dos mediciones.

$$
\begin{gathered}
v=\frac{\mathrm{U} s}{0 t} \\
\left.\Delta s=M_{1} \mathbf{d} v_{\text {sonido }} \cdot \frac{t_{\text {sonido }}}{2}\right) \\
-M_{2}\left(v_{\text {sonido }} \cdot \frac{t_{\text {sonido }}}{2}\right)
\end{gathered}
$$

Donde $M_{1}$ y $M_{2}$ son las distancias tomadas desde el quadrotor hasta el objeto obstáculo.

$v$, es la velocidad del quadroter en función de la distancia recorrida tomada de las mediciones de los sensores ultrasonido.

\section{Sensor Inercial}

Los sensores inerciales miden la aceleración y velocidad angular respecto a un eje de referencia. Está compuesto por acelerómetros, giróscopos y magnetómetros. Los acelerómetros miden la aceleración lineal respecto a los ejes $\left(\begin{array}{lll}x & y & z\end{array}\right)$, los giróscopos miden la velocidad angular respecto a los ejes $\left(\begin{array}{lll}x & y & z\end{array}\right)$ y los 
magnetómetros dan información acerca del norte magnético en grados.

El sensor seleccionado es un GY-85 IMU; que a su vez integra tres sensores (14-16):

- $\quad$ Acelerómetro de 3 ejes,

- $\quad$ Giroscopio de 3 ejes

- $\quad$ Sensor de campo magnético de 3ejes.

Las referencias de los integrados son:

- $\quad$ ADXL345 (Acelerómetro)

- HMC5883L (sensor de campo magnético)

- $\quad$ ITG3205 (Giroscopio)

\section{E. Estimación del angulo de inclinación}

Las ecuaciones que permiten transformar los datos de aceleración de cada uno de los ejes, en el ángulo de inclinación del UAV, se describen en la ecuación \# (17)

$$
\begin{array}{r}
\theta=\arctan \left(\frac{A x}{\sqrt{A y^{2}+A z^{2}}}\right) \\
\psi=\arctan \left(\frac{A y}{\sqrt{A x^{2}+A z^{2}}}\right) \\
\Phi=\left(\frac{\sqrt{A x^{2}+A y^{2}}}{z}\right)(7)
\end{array}
$$

Donde:

$$
\begin{aligned}
& A_{x}=F_{x} \\
& A_{y}=F_{y} \\
& A_{z}=F_{z}
\end{aligned}
$$

Estos son datos entregados por el acelerómetro.

\section{F. Estimación de las mediadas de distancia}

La estimación de las medidas tomadas con cada uno de los sensores, ya sean de distancia o inerciales, no debe ser ingresada directamente al controlador, la razón, son las posibles perturbaciones relacionadas con el ambiente, presión atmosférica o aceleración (Hafner et al., 2010) de esta forma se usa un filtro de Kalman para fusionar dos canales sensoriales para una sola estimación de altitud, de distancia y acelera- ción respecto a objetos circundantes, suavizando las perturbaciones inesperadas mediante determinando el error medio cuadrático (Técnico, 2003) (18).

\section{G. Filtro de Kalman}

El filtro de Kalman es un algoritmo que permite identificar el estado oculto (no medible) de un sistema dinámico lineal, pero sirve además cuando el sistema está sometido a ruido blanco aditivo. El filtro de Kalman es capaz de escoger la de forma óptima cuando se conocen las varianzas de los ruidos que afectan al sistema. El filtro de Kalman es un algoritmo recursivo y óptimo de procesamiento de datos, el cual tiene varias aplicaciones, entre ellas, es útil para la estimación de modelos auto regresivos (19), la estimación de modelos con parámetros que cambian en el tiempo, la estimación de modelos con componentes no observables. Además puede estimar sistemas dinámicos especificados en la forma de estado-espacio (State-Space). Los modelos estado-espacio son esencialmente una notación conveniente para abordar el manejo de un amplio rango de modelos de series de tiempo (11).

\section{H. Control de velocidad mediante logica difusa}

El controlador de lógica difusa se diseñó para automatizar cómo un experto humano el control de velocidad en función de la distancia hacia un obstáculo, tomando como referencia el ingreso en un área que presenta riesgo de colisión. El control difuso requerido para el sistema es de siete entradas y dos salidas. Los datos de entrada provienen de los cuatro sensores ultrasonido que entregan la distancia hasta los objetos circundantes, además tomando la distancia con un mismo sensor en la dirección del movimiento, se determina la velocidad del UAV, la cual se ingresa como una quinta entrada al controlador difuso; las otras dos entradas corresponden a los ángulos de inclinación obtenidos a partir de la lectura del acelerómetro. Las salidas del controlador difuso se refieren a los ángulos (Pitch y Roll), con su respectiva corrección para evitar la colisión con el obstáculo. En la tabla I se describen las funciones de membrecía para la entrada y la salida (20). 
Tabla 1, funciones de membrecía

\begin{tabular}{lll}
\hline \multicolumn{1}{c}{ Input Sensores } & \multicolumn{1}{c}{ Tipo } & \multicolumn{2}{c}{ Rango } \\
\hline Cerca & Triangular & {$\left[\begin{array}{lll}0.5 & 1.5 & 2.5\end{array}\right]$} \\
Cerca Riesgo & Triangular & {$\left[\begin{array}{lll}-0.5 & 0.5 & 1.5\end{array}\right]$} \\
Medio & Triangular & {$\left[\begin{array}{lll}1.5 & 2.5 & 3.5\end{array}\right]$} \\
Lejos & Triangular & {$\left[\begin{array}{llll}2.5 & 3.5 & 4.5\end{array}\right]$}
\end{tabular}

\section{Input Velocidad $\mathrm{m} / \mathrm{s}$}

$\begin{array}{lllr}\text { Baja } & \text { Triangular } & {\left[\begin{array}{llr}0 & 1 & 2\end{array}\right]} \\ \text { Media } & \text { Triangular } & {\left[\begin{array}{llr}1 & 2.25 & 3.5\end{array}\right]} \\ \text { Media Alta } & \text { Triangular } & {\left[\begin{array}{llr}2.5 & 3.75 & 5\end{array}\right]}\end{array}$

\section{Input Pitch, Roll}

\begin{tabular}{|c|c|c|c|}
\hline Baja Inclinación & Triangular & {$[-45$} & -45 \\
\hline Media Inclinación & Triangular & {$[-35$} & 0 \\
\hline Alta Inclinación & Triangular & {$[10$} & 45 \\
\hline
\end{tabular}

\section{Output Pitch, Roll}

\begin{tabular}{llll} 
Baja Inclinación & Triangular & {$\left[\begin{array}{ccc}-45 & -45 & -9\end{array}\right]$} \\
Media Inclinación & Triangular & {$\left[\begin{array}{ccc}-35 & 0 & 35\end{array}\right]$} \\
Alta Inclinación & Triangular & {$\left[\begin{array}{lll}10 & 45 & 45\end{array}\right]$} \\
\hline
\end{tabular}

Para el diseño del controlador difuso se utilizó el toolbox de Matlab, en el cual se construyeron los conjuntos difusos, las reglas que las relacionan y las salidas de ángulo, Pitch y Roll. Estas salidas sirven como insumo a un segundo controlador quien entrega los valores exactos de potencia de los motores o en caso de una plataforma de ala fija entrega el movimiento de los alerones (21).

\section{RESULTADOS Y ANÁLISIS}

En esta sección se presentan los resultados de la caracterización del sensor ultrasonido usado en el mapeo, además de la implementación del controlador inteligente que relaciona y estima la velocidad a partir de la lectura de los sensores de distancia respecto a un objeto que se encuentra en el camino.

\section{A. Datos experimentales}

Los resultados experimentales de la caracterización del sensor de distancia por ultrasonido de referencia HC-SR04 se presentan en la tabla 2.

En las pruebas del sistema, se utilizó un obstáculo, ubicado a $2 \mathrm{mt}$, del sensor. El sensor y el obstáculo se montaron con soportes especiales de precisión sobre una mesa para aplicaciones electrónicas; con el fin de obtener la mayor exactitud en la toma de los datos mencionados. Con la ayuda de un sistema de medición de temperaturas, se tomo la temperatura promedio entre ambos obstáculos, realizando un Barrido de temperatura desde $17^{\circ} \mathrm{C}$ (Temperatura ambiente) hasta $33^{\circ} \mathrm{C}$.

Los resultados nos permiten obtener la ecuación lineal que relaciona la velocidad del sonido y la temperatura en el lugar de pruebas para el sensor propuesto, figura 2 .

$$
v_{\text {sonido }}=0.8008 * T+328.27
$$

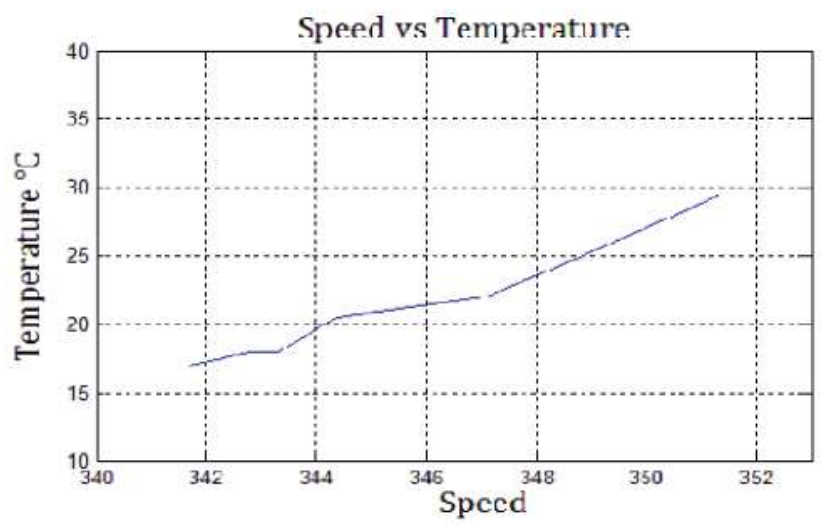

Figura 3, respuesta de la velocidad del sonido en función de la temperatura 
Tabla 2. Estadística sensor ultra sonido

\begin{tabular}{cccccc}
\hline Distancia & mt & Tiempo & sg & Velocidad & mt/sg \\
\hline Media & 2 & Media & 0,00578629 & Media & 345,5625 \\
Error típico & 0 & Error típico & $4,17 \mathrm{E}-06$ & Error típico & 0,24915497 \\
Mediana & 2 & Mediana & 0,0058 & Mediana & 344,828 \\
Moda & 2 & Moda & 0,00585 & Moda & 347,1 \\
Desviación estándar & 0 & Desviación estándar & $6,04 \mathrm{E}-05$ & Desviación estándar & 3,6105985 \\
Varianza de la muestra & 0 & Varianza de la muestra & $3,65 \mathrm{E}-09$ & Varianza de la muestra & 13,0364 \\
Curtosis & 0 & Curtosis & $-1,25297118$ & Curtosis & $-1,2422$ \\
Coeficiente se asimetría & 0 & Coeficiente se asimetría & $-0,414701648$ & Coeficiente se asimetría & 0,403362 \\
Rango & 0 & Rango & 0,00024 & Rango & 14,277 \\
Mínimo & 2 & Mínimo & 0,00564 & Mínimo & 340,136 \\
Máximo & 2 & Máximo & 0,00588 & Máximo & 354,412 \\
Coeficiente de variación & 0 & Coeficiente de variación & 0,010444759 & Coeficiente de variación & 0,0104484 \\
\hline
\end{tabular}

Los resultados obtenidos para el sensor HC-SR04, muestran una precisión menor a $0.5 \mathrm{~cm}$ en la medición de distancia del UAV hacia el objeto que se presenta como obstáculo, a la vez que nos permite determinar de forma confiable la velocidad de aproximación del UAV. De esta forma el controlador difuso pueda entregar los datos de corrección para evitar la colisión.

\section{B. Control anticolisión difuso}

En esta sección son mostrados los resultados del controlador difuso para prevenir colisiones en entornos cerrados. En la Ilustración III, se presenta la respuesta del controlador difuso ante el ingreso del UAV en el área de riesgo de colisión. Está área de colisión está definida por la distancia máxima que puede medir el sensor Ultrasonido seleccionado. Para la referencia HC-SR04, es de cuatro metros, más la corrección por temperatura antes hallada en la ecuación 8 .

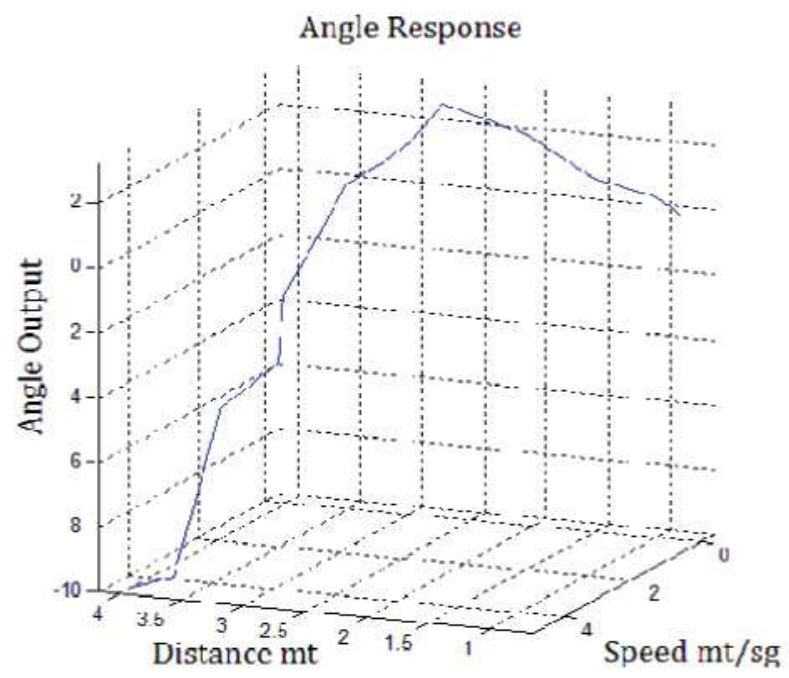

Figura 4, Ángulo de salida en función de la velocidad y la cercanía al obstáculo
Los resultados muestran que mientras el UAV se encuentre fuera del área de colisión, el controlador se encuentra desactivado, pero a medida que el vehículo ingresa en el área de riesgo el controlador evalúa la velocidad de aproximación, la inclinación angular (Pitch, Roll), entregando el Angulo de salida corregido (Pitch, Roll) que debe tomar el UAV para reducir la velocidad lineal de desplazamiento y prevenir la colisión.

En la figura 4, se muestra la plataforma de pruebas usada, la cual se diseño para este Proyecto. La plataforma consta de un Sistema Arduino FIO, el IMU GY-85, cuatro sensores HC-SR04 y la comunicación se realiza mediante módulos Zigbee, los cuales se conectan al controlador fuzzy ejecutado desde Matlab.

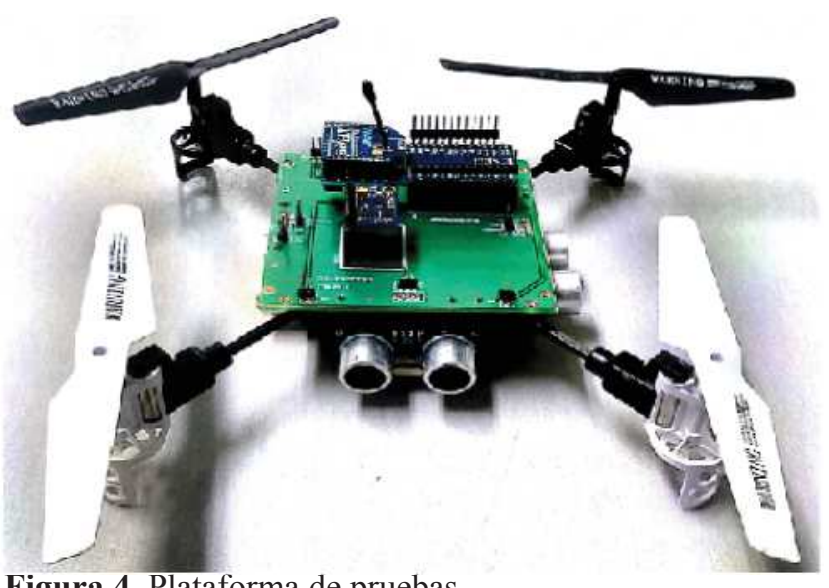

Figura 4, Plataforma de pruebas

\section{CONCLUSIONES}

Presentando el prototipo de vehículo aéreo utilizado para la realización de las pruebas reales, se demuestra que es posible implementar un UAV completamente 
funcional y de bajo costo y bajo procesamiento, siendo implementado autónomamente con el controlador presentado en esta propuesta. Aunque el controlador es solo una medida de aseguramiento e independencia del vehículo contra colisiones, también se hace posible incorporar la navegación controlada por medio de otros dispositivos remotos sin perder autonomía ni prioridad del controlador difuso.

Los resultados también sugieren claramente que el controlador difuso provee el procesamiento de las señales necesarias obteniendo resultados satisfactorios con respecto prevención de colisiones, logrando detener el vehículo Aéreo no tripulado antes de colisionar con el obstáculo.

El sistema propuesto para prevención de colisiones puede ser implementado tanto en UAVs guiados a control remoto, como en vehículos completamente autónomos, ya que se propone como un sistema a bajo nivel, donde entra en funcionamiento cuando el UAV se aproxima a un obstáculo a una velocidad que presenta riesgo de colisión, de esta forma el controlador cambia el ángulo de inclinación para disminuir la velocidad de aproximación hasta detener el UAV.

Aunque el controlador presenta algunas limitantes con respecto al procesamiento de los diferentes datos obtenidos mediante la cantidad de sensores implementados; como trabajo futuro será posible utilizar procesadores sobre sistemas embebidos para realizar procesamiento en la board directamente sobre el vehículo aéreo no tripulado

\section{AGRADECIMIENTOS}

Este trabajo está enmarcado en labores de investigación del grupo de investigación en Automática, electrónica y Ciencias Computacionales, del Centro de Investigación del Instituto Tecnológico Metropolitano, Medellín, Colombia. Con el proyecto de investigación P14101.

\section{BIBLIOGRAFÍA}

1. Sáenz S, Aguirre J, Zuluaga-- J, Hazbon O, Bolivariana UP, Paulo UDS, et al. Diseño Detallado de un Vehículo Aéreo No Tripulado Multipropósito de Bajo Costo para su Operación en Colombia. :1-9.

2. Quadcopter : Construcción, control de vuelo y navegación GPS Autor : Eduardo Parada Pardo Director : 2012 ;

3. Morgenthaler S, Braun T, Zhao Z, Staub T, Anwander M. UAVNet: A mobile wireless mesh network using Unmanned Aerial Vehicles. 2012 IEEE Globecom Work [Internet]. Ieee; 2012 Dec;1603-8. Available from: http://ieeexplore.ieee.org/lpdocs/epic03/wrapper.htm?arnumber $=6477825$

4. Muller M, Lupashin S, D'Andrea R. Quadrocopter ball juggling. 2011 IEEE/RSJ Int Conf Intell Robot Syst [Internet]. Ieee; 2011 Sep;5113-20. Available from: http://ieeexplore.ieee.org/lpdocs/epic03/ wrapper.htm?arnumber $=6094506$

5. Nagai M, Shibasaki R, Kumagai H, Ahmed a. UAV-Borne 3-D Mapping System by Multisensor Integration. IEEE Trans Geosci Remote Sens [Internet]. 2009 Mar;47(3):701-8. Available from: http:// ieeexplore.ieee.org/lpdocs/epic03/wrapper.htm?arnumber $=4783021$

6. Go J. Unscented Kalman Filter for an orientation module of a quadrotor mathematical model. 2013;

7. Elizabeth FQ, Schoellig AP, Wiltsche C, Andrea RD. 2012 American Control Conference FeedForward Parameter Identification for Precise Periodic Quadrocopter Motions. 2012;

8. Hafner V V, Bachmann F, Berthold O, Schulz M, Müller M. An autonomous flying robot for testing bio-inspired navigation strategies Quadrocopter platform Simulation of the Multicopter platform. 2010;1145-51.

9. Puls T, Hein a. 3D trajectory control for quadrocopter. 2010 IEEE/RSJ Int Conf Intell Robot Syst [Internet]. Ieee; 2010 Oct;640-5. Available from: http://ieeexplore.ieee.org/lpdocs/epic03/wrapper. htm?arnumber $=5650249$

10. Yue $\mathrm{C}, \mathrm{Yu} \mathrm{H}, \mathrm{Xu}$ J. Design of quartz accelerometer signal acquisition system. 2011 IEEE Int Conf Mechatronics Autom [Internet]. Ieee; 2011 Aug;1578-82. Available from: http://ieeexplore.ieee.org/ lpdocs/epic03/wrapper.htm?arnumber $=5986229$ 
11. Eckert J, German R, Dressler F, Member S. An Indoor Localization Framework for Four-Rotor Flying Robots Using Low-Power Sensor Nodes. 2011;60(2):336-44.

12. Vojtech Š, Dušan N, Jozef H, Definitions A. Calculation of Robot Position Utilizing Accelerometers in Non-inertial Frame of Reference. 2012;373-6.

13. Puls T, Hein a. Outdoor position estimation and autonomous landing algorithm for quadrocopters using a wireless sensor network. 2010 IEEE/ASME Int Conf Adv Intell Mechatronics [Internet]. Ieee; 2010 Jul;285-90. Available from: http://ieeexplore.ieee.org/lpdocs/epic03/wrapper.htm?arnumber=5695747

14. Parameter E. Ultrasonic Ranging Module HC - SR04. 2011;3-5.

15. Range F. 3-Axis Digital Compass IC. 2011;1-18.

16. Ave B, Number D, Date R. ITG-3205 Product Specification. 2010;1(408):1-38.

17. Yao X, Sun G, Lin W-Y, Chou W-C, Lei KF, Lee M-Y. The design of an in-line accelerometer-based inclination sensing system. 2012 IEEE Int Symp Circuits Syst. Ieee; 2012 May;333-6.

18. Razak NA, Hashim N, Arshad M, Adnan R, Misnan MF, Thamrin NM, et al. A Study of Kalman 's Filter in Embedded Controller for Real-Time Quadrocopter Roll and Pitch Measurement. 2012;23-5.

19. Técnico I. Uso del filtro de kalman para estimar la tendencia de una serie. 2003;

20. Juriquilla C. artículo invitado La Lógica difusa en ingeniería : PrinciPios, aPLicaciones y futuro conceptos y aplicaciones. 2006;24(2):87-107.

21. Olivera S, Asesor AG. Sistema de Control de un Vehículo Aéreo Autónomo Pequeño. 2002; 